Cahiers $d u$ MONDE RUSSE

\section{Cahiers du monde russe}

Russie - Empire russe - Union soviétique et États indépendants

$61 / 3-4 \mid 2020$

Écritures visuelles, sonores et textuelles de la justice

\title{
Aminat CHOBOKAEVA, Cloé DRIEU et Alexander MORRISON, éds. , The Central Asian Revolt of 1916. A Collapsing Empire in the Age of War and Revolution
}

\section{Aude-Cécile Monnot}

\section{(2) OpenEdition}

\section{Édition électronique}

URL : https://journals.openedition.org/monderusse/12193

DOI : 10.4000/monderusse. 12193

ISSN : $1777-5388$

Éditeur

Éditions de l'EHESS

\section{Édition imprimée}

Date de publication : 1 juillet 2020

Pagination : $546-549$

ISBN : 978-2-7132-2832-2

ISSN : $1252-6576$

\section{Référence électronique}

Aude-Cécile Monnot, " Aminat CHOBOKAEVA, Cloé DRIEU et Alexander MORRISON, éds. , The Central Asian Revolt of 1976. A Collapsing Empire in the Age of War and Revolution », Cahiers du monde russe [En ligne], 61/3-4 | 2020, mis en ligne le 01 juillet 2020, consulté le 11 septembre 2022. URL : http:// journals.openedition.org/monderusse/12193; DOI : https://doi.org/10.4000/monderusse.12193 


$\begin{array}{cccccccccccccccccccccc}\text { I } & \mathrm{D} & \mathrm{E} & \mathrm{N} & \mathrm{T} & \mathrm{I} & \mathrm{T} & \mathrm{E} & \mathrm{S} & & \mathrm{N} & \mathrm{A} & \mathrm{T} & \mathrm{I} & \mathrm{O} & \mathrm{N} & \mathrm{A} & \mathrm{L} & \mathrm{E} & \mathrm{S} & \\ & & \mathrm{E} & \mathrm{M} & \mathrm{P} & \mathrm{I} & \mathrm{R} & \mathrm{E} & \mathrm{S} & & & \mathrm{R} & \mathrm{E} & \mathrm{G} & \mathrm{I} & \mathrm{O} & \mathrm{N} & \mathrm{S} & & \end{array}$

Aminat CHOBOKAEVA, Cloé DRIEU et Alexander MORRISON, éds. The Central Asian Revolt of 1916

\section{A Collapsing Empire in the Age of War and Revolution}

Manchester : Manchester University Press, 2019, 360 p.

Cet ouvrage collectif est une contribution essentielle à l'historiographie de la révolte de 1916 en Asie Centrale. Cette vague de révoltes survint durant l'été 1916 après la publication du décret impérial du 25 juin/7 juillet de conscription des inorodcy [indigènes], la population centrasiatique, dans des bataillons de travail. Ce mouvement débuta dans la région de Hudžand, au nord du Tadjikistan, puis gagna en intensité à Džizak (actuel Ouzbékistan), puis au Ferghana. En août, des soulèvements violents opposèrent les populations kazakhes et kirghizes aux colons russes dans la région du Semireč'e. Enfin, en septembre 1916, la révolte gagna les steppes du Nord Kazakhstan, alors même que des bataillons de l'armée impériale engageaient avec force et violence le processus de répression des soulèvements. (150 000 morts côté centrasiatique, 3000 colons slaves, p. 20).

Le renouvellement historiographique suscité par les centenaires de la Grande Guerre et des révolutions de 1917 avait jusqu'alors, à de rares exceptions près, peu nourri les études sur les révoltes de 1916 mentionnées dans ce volume. L'historiographie de 1916 était encore l'héritière des analyses historiques de l'époque soviétique. Celles-ci ont, certes, fourni des données et des sources précieuses ${ }^{1}$, mais elles ont aussi façonné une lecture très idéologique de la révolte de 1916. En effet, comme analysé de manière parfaitement didactique dans l'introduction de l'ouvrage, l'historiographie soviétique avait presque érigé les révoltes de 1916 en prélude de la révolution de 1917, voyant dans les mobilisations en 1916 la preuve 
d'une tradition révolutionnaire en Asie centrale et de l'iniquité du régime tsariste (p. 4). L'ouvrage se propose donc de renouveler l'historiographie sur cette révolte et il y parvient en déployant au fil des chapitres une analyse précise et subtile, non pas de la révolte, mais des révoltes de 1916. L'ouvrage s'attache particulièrement à analyser les causes structurelles et conjoncturelles de ces révoltes, les acteurs qui participèrent à ces mouvements et leurs motivations dans diverses configurations régionales. L'analyse porte également sur les dynamiques de la violence et l'importance du contexte de guerre et ouvre un champ d'analyse sur la mémoire de ces révoltes.

Avant d'analyser la structure et l'argumentaire des différentes contributions, il faut souligner l'intérêt disciplinaire de cet ouvrage par-delà la question des révoltes de 1916. Grâce aux collaborations entre chercheurs anglo-saxons, français, russes et centrasiatiques, cette étude parvient à établir un dialogue entre différentes cultures historiographiques et des sources secondaires multilingues. Grâce à cette masse critique, l'historiographie de la période soviétique est déconstruite et fournit des sources utiles au déploiement d'une analyse nuancée des révoltes de 1916. Il faut également souligner la place accordée aux sources vernaculaires, particulièrement les sources orales - des poèmes, chansons, prose en kazakh, en kirghiz et en ouïghour -, qui permettent aux auteurs, (Ablet Kamalov, Jipar Duishembieva, Daniel Prior et Danielle Ross), d'intégrer les récits et les perspectives d'acteurs centrasiatiques jusqu'alors peu étudiés.

L'ouvrage décline l'analyse des révoltes de 1916 en plusieurs thématiques qui sont autant de questions de recherche sur les causes des révoltes, les dynamiques et les acteurs de la violence, et enfin la mémoire des révoltes de 1916. Dans ce parcours thématique, plusieurs chapitres adoptent des perspectives différentes et complémentaires: aux analyses régionales (Akmal Bazarbaev et Cloé Drieu, Aminat Chokobaeva) répondent des analyses globales intégrant les révoltes de 1916 dans le cadre de la Première Guerre mondiale (Niccolò Pianciola et Alexander Morrison). Les chapitres varient quant au cadre temporel, à des analyses conjoncturelles (Oybek Mahmudov, Jörn Happel) répondent des analyses sur le temps long des révoltes anticoloniales (Isabelle Ohayon et Xavier Hallez).

Les premiers chapitres analysent les causes des révoltes de 1916. L'ensemble des contributions s'accordent sur les causes structurelles - à savoir les clivages socioéconomiques entre colons slaves et populations indigènes engendrés par la colonisation et l'appropriation de terres -, et le facteur déclencheur du décret impérial de mobilisation en juin 1916 (Tatiana Kotiukova). Cependant, le premier chapitre de Tomohiko Uyama soutient que ces facteurs ne suffisent pas à expliquer la spécificité géographique et temporelle de la révolte, en Asie centrale et précisément en 1916. Son analyse plurifactorielle montre comment le contexte de guerre transforma la « crise coloniale latente en une crise explicite » (p. 40). La mobilisation des populations centrasiatiques dans des travaux d'arrière requérait un effort de mobilisation que la faiblesse de l'administration impériale en Asie centrale, aggravée par la mobilisation des forces armées pour la guerre et la méfiance réciproque entre colons et indigènes, rendit impossible. Dans cette analyse des causes, les contextes 
régionaux sont signifiants et soulignent des dynamiques spécifiques. Ainsi Akmal Bazarbaev et Cloé Drieu montrent que les pressions économiques immédiates de la guerre, notamment sur le prix des céréales, ont déclenché les révoltes au Džizak (chap. 3).

Les dynamiques du soulèvement, qui ont fait suite à l'ordre de mobilisation des populations d'Asie centrale, et les dynamiques de violence (p. 154-158) traduisent les clivages et la méfiance entre l'appareil colonial et les populations centrasiatiques évoqués plus haut. Des rumeurs, l'incompréhension par les administrateurs coloniaux des révoltes, interprétées, sans fondement souligne Oybek Mahmudov, en termes d' " irrationalité » et de « fanatisme religieux » (p. 117), alimentent une panique et justifient une répression brutale. L'ampleur de ces violence s'inscrit dans une histoire globale de la Grande Guerre, elle traduit la « brutalisation des sociétés » et le rôle des soldats démobilisés et rapatriés du front comme vecteurs de la violence de guerre en contexte impérial ${ }^{2}$. Niccolò Pianciola démontre que les soldats venus du front anatolien réitèrent contre les populations kazakhes et kirghizes du Semireč'e des pratiques « éliminationistes »d'épuration ethnique conduites sur le front ottoman contre les populations civiles kurdes et turques. La violence de la répression en 1916 resurgit d'ailleurs pendant la période révolutionnaire dès 1917 portée par des groupes paramilitaires et des soviets paysans contre les populations du Turkestan et du Semireč'e. Ici, la violence de 1916 s'inscrit dans un cadre d'analyse globale d'un « continuum de crises » de l'impérialisme, défini entre autres par Peter Holquist et Joshua Sanborn, ${ }^{3}$ qui s'ouvre en 1914 et se poursuit jusqu'en 1920.

Cependant, toutes les contributions n'adoptent pas ce cadre d'analyse globale et plusieurs chapitres insistent au contraire sur la spécificité de 1916 et ses dynamiques régionales propres, y compris dans le temps long, voire « dans l'ombre de la conquête » du Turkestan pour Ian W. Campbell. Le chapitre de Xavier Hallez et Isabelle Ohayon explore ainsi un continuum de crises spécifiquement kazakhes, en situant 1916 dans une série de révoltes de 1837-1847 à 1929-1931. Ce chapitre suggère l'émergence d'un modèle de résistance anticoloniale (un « modus operandi », p. 272) articulé autour de deux figures politiques, celle du batyr (figure héroïque kazakhe, devenue un «bandit social $»^{4}$ ) et celle du khan. Cet héritage des luttes politiques, leurs motivations et leurs outils d'action, pourraient d'ailleurs faire l'objet de recherches sur d'autres régions de l'Asie centrale, particulièrement au Turkestan.

Les derniers chapitres de l'ouvrage accordent une place nécessaire aux perspectives et aux discours de l'intelligentsia centrasiatique et analysent la mémoire populaire de 1916. Ces travaux soulignent que la postérité de ces événements est indissociable des processus de construction nationale en Asie centrale pendant la période soviétique (p. 329)

Pour conclure, la variété des contributions et des thématiques de recherche constitue la principale force de cet ouvrage. Elle est aussi sa complexité pour le lecteur qui aborderait la révolte de 1916 par cette analyse dense mêlant l'étude des causes, des dynamiques et de la mémoire à une réflexion critique sur 
l'historiographie soviétique et contemporaine. En effet, un débat historiographique se tisse au fil des chapitres qui se répondent, voire même se nuancent les uns les autres, sur le rôle des administrateurs impériaux indigènes pendant les révoltes ; il forme aussi sur le cadre d'analyse privilégié pour comprendre les révoltes de 1916, soit comme un événement dans la crise impériale globale de la Grande Guerre et de la révolution, soit au contraire comme une mobilisation anticoloniale avec ses propres dynamiques régionales et locales. L'ouvrage ne tranche pas et les éditeurs soulignent délibérément la portée des révoltes de 1916 pour trois champs historiographiques, celui des révoltes anticoloniales, celui de la Grande Guerre et enfin, celui de l'Empire russe. L'ouvrage livre ainsi un agenda de recherche pour de futures contributions et une analyse essentielle et polyphonique sur les révoltes de 1916 en Asie centrale.

1 - À titre d'exemples, G.I. Broido, Vosstanie Kirgiz v 1916g. Moe pokazanie prokuroru taškentskoj sudebnoj palaty, dannoe 3-go Sentjabrja 1916g [La révolte des Kirghiz en 1916. Mon témoignage au procureur du tribunal de Taškent, 3 sept. 1916], M. : Naučnaja Associacija Vostokovedenija pri TsIK SSR, 1925 ; T. Ryskulov, "Vosstanie uzemtsev Turkestana v 1916 godu [Le soulèvement des indigènes du Turkestan en 1916]", Očerki revoljucionnogo dviženija v Srednej Azii. Sbornik statej [Essai sur le mouvement révolutionnaire en Asie centrale. Recueil de documents], M., 1926, p. 46-122.

2 - George L. Mosse, De la Grande Guerre au totalitarisme, la brutalisation des sociétés européennes, $1^{\text {re }}$ éd., Oxford University Press, 1990.

3 - Peter Holquist, Making War, Forging Revolution: Russia's Continuum of Crisis, Cambridge : Harvard University Press, 2002 ; Joshua Sanborn, Imperial Apocalypse : The Great War and the Destruction of the Russian Empire, Oxford University Press, 2013, p. 175-183.

4 - Eric Hobsbawm, Bandits, New York: Patheon Books, 1981.

Aude-Cécile Monnot

Centre d'Histoire, SciencesPo Paris 